SECTION 4. Computer science, computer engineering and automation.

Sizyakin Roman Alekseyevich

a master's student of Department «Radioelectronic systems» Research Institute «Digital signal processing and computer vision» Don state technical University, Russia Voronin Vyacheslav Vladimirovich candidate of technical Sciences, associate Professor,

Department «Radioelectronic systems»

Research Institute «Digital signal processing and computer vision»

Don state technical University, Russia

Marchuk Vladimir Ivanovich

doctor of technical Sciences, Professor, Department «Radioelectronic systems» Research Institute «Digital signal processing and computer vision» Don state technical University, Russia

Fisunov Aleksandr Vladimirovich

postgraduate student, Department «Radioelectronic systems» Research Institute «Digital signal processing and computer vision» Don state technical University, Russia

\title{
DEFECTS DETECTION ON THE IMAGE
}

In the paper we use the method of texture analysis in relation to the problem at hand, which is based on the analysis of the convolution of the original image with the kernel Gabor.

Keywords:defects, image analysis.

\section{ОБНАРУЖЕНИЕ ДЕФЕКТОВ НА ИЗОБРАЖЕНИИ}

В работе предлагается использовать метод текстурного анализа применительно $\kappa$ решаемой задаче, который основан на анализе результата свертки исходного изображения с ядром Габора.

Ключевые слова:дефекты, изображение, анализ.

На практике встречается большое количество изображений, которые имеют дефекты в виде трещин и царапин. Многие фотографии являются уникальными и в некоторых случаях единственными сведениями об отдельных элементах прошлого. Оценивание истинных значений пикселей изображений в той или иной степени необходимо в большинстве задач цифровой обработки изображений. Методы восстановления двумерных 
сигналов находят свое применение в задачах обработки архивных документов в виде изображений, имеющих различные искажения, например: царапины, пятна, пыль, не нужные надписи, линии сгиба. Изображения с дефектами могут быть получены с камер служб по обслуживанию дорог, фотографий муниципальных управлений и т.д. Задача автоматизированного обнаружении трещин дорожного покрытия на изображении схожа с задачей обнаружения царапин на архивных фотографиях, так как трещины дорожного покрытия представляют собой в большинстве случаев тонкую, кривую линию темного цвета (рис. 1а), в то время как трещины на архивных изображениях имеют схожую структуру, однако цвет имеет преимущественно светлые тона (рис. 1б).

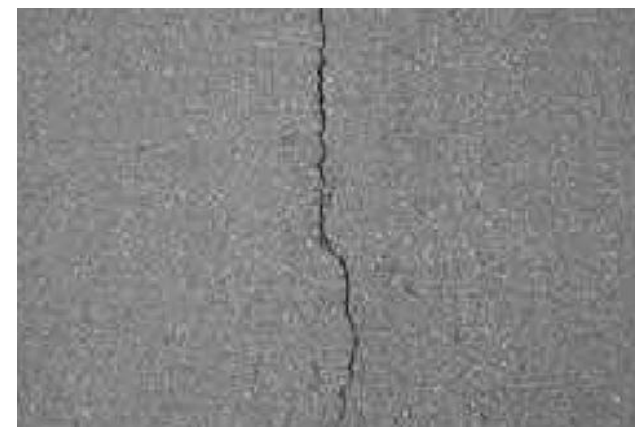

a)

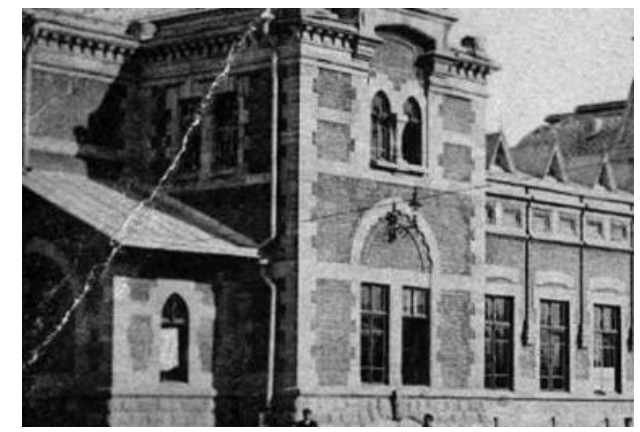

б)

Рисунок 1 - Пример дефектов на изображении.

В настоящее время существует большое количество методов автоматического детектирования дефектов на изображении. Самые первые и наиболее простые в реализации алгоритмы относятся к классу пороговой обработки [1, с. 402-418,]. Основным недостатком данных методов является зависимость количества ложных срабатываний от уровня порогового значения. Другим классом детекторов является морфологическая обработка изображений [2, с. 154-157]. Для обнаружения дефектов на фотодокументах при условии предварительной фильтрации используются морфологические операции для обнаружения светлых и темных трещин соответственно [3]. Также возможно использование различных комбинаций морфологических операций. Данный класс детекторов имеет в результирующей маске дефектов меньше ложных срабатываний, чем детекторы, основанные на пороговой обработке, но, тем не менее, эффективность также зависит от априорно заданных параметров. В статье [4] авторами предлагаются методы обнаружения дефектов путем использования классификатора с предварительным обучением с учителем. Данный класс детекторов частично устраняет недостатки описанных ранее методов, так как не нуждается в задании начальных параметров, но для корректной работы 
необходимо время на обучение алгоритма, что в свою очередь может ограничить круг решаемых задач.

В литературе также известно большое количество методов, которые не относятся к описанным выше классам - методы, основанные на анализе контуров трещин, методы адаптивной фильтрации и методы на основе Марковской модели сегментации [5, 6, с.50-53, 7, с.16-20, 8, с.46-51]. В большей степени все описанные методы применяются к задаче обнаружения трещин на дорожном покрытии и плохо адаптированы к поиску царапин на архивных фотодокументах.

Целью данной работы является обнаружение на архивных изображениях дефектов, вызванных отслоением фотографического слоя, с целью дальнейшего их устранения в автоматическом режиме.

Изображение рассматривается как упрощенная математическая модель, которая представляет собой двумерную дискретную последовательность $Y_{i, j}, i=\overline{1, N}, j=\overline{1, M}$, вида:

$$
Y_{i, j}=\left(1-d_{i, j}\right) \cdot S_{i, j}+d_{i, j} \cdot c_{i, j},
$$

где $Y_{i, j}$ - наблюдаемое изображение, $S_{i, j}$ - оригинальное (неповрежденное) изображение, $d_{i, j}$ - бинарная маска области с искаженными значениями ( 1 - соответствует искаженным пикселям, 0 соответствует не искаженным пикселям), $c_{i, j}$ - искаженные значения пикселей.

В работе предлагается использовать метод текстурного анализа применительно к решаемой задаче, который основан на анализе результата свертки исходного изображения $Y_{i, j}$ с ядром Габора [9].

Функция Габора имеет следующий вид:

$$
g(x, y ; \lambda, \theta, \psi, \sigma, \gamma)=\exp \left(-\frac{x^{\prime 2}+\gamma^{2} y^{\prime 2}}{2 \sigma^{2}}\right) \cos \left(2 \pi \frac{x^{\prime}}{\lambda}+\psi\right),
$$

где $\quad x^{\prime}=x \cos \theta+y \sin \theta, \quad y^{\prime}=-x \sin \theta+y \cos \theta, \quad \lambda \quad$ - длина волны множителя-косинуса, $\theta$ - ориентация нормали параллельных полос функции Габора в градусах, $\psi$ - сдвиг фаз в градусах, $\gamma$ - коэффициент сжатия, характеризующий эллиптичность функции.

На основе проведенных исследований выбраны следующие параметры функции Габора: две ориентации нормали $\theta$ равные $90^{\circ}$ и $180^{\circ}$, окно размером $5 \times 5$ пикселей. 


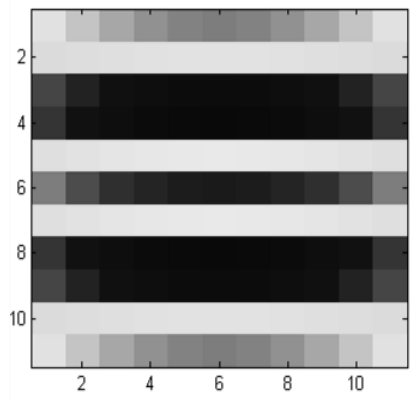

a)

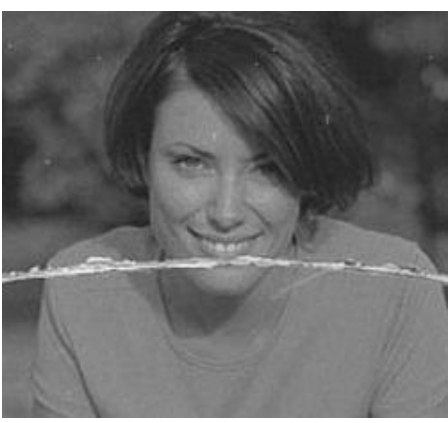

б)

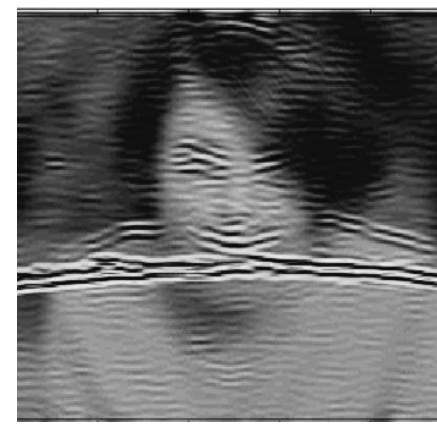

B)

Рисунок 2 - Ядро Габора и результат свертки

Выбор окна размером $5 \times 5$ обусловлен тем, что трещины на изображении имеют структуру тонкой линии, а при увеличении размера данного параметра появляются искажения в виде дополнительных колебаний вокруг предполагаемых дефектов, что уменьшает вероятность правильного обнаружения. На рисунке 2 представлен пример, иллюстрирующий данный эффект при окне размером $11 \times 11$ (а - ядро Габора; б - исходное изображение; в - результат свертки). Параметр $\theta$ равный $90^{\circ}$ и $180^{\circ}$ обеспечивает наибольшую универсальность, что в большинстве случаев является достаточным для детектирования дефектов на изображении.

После нахождения свертки $G_{i, j}$ к ней применяется операция бинаризации, необходимая для того что бы выделить области, получившие наибольший отклик. В результате получается маска $M_{i, j}$. Поскольку в данной работе используется ядро размером $5 \times 5$ пикселей, фильтр имеет только один экстремум, в результате чего большой отклик получают и квазистационарные светлые области. Пример таких областей представлен на рисунке 3. Соответственно использование результирующей бинаризованной функции отклика как маски дефектов не представляется возможным.

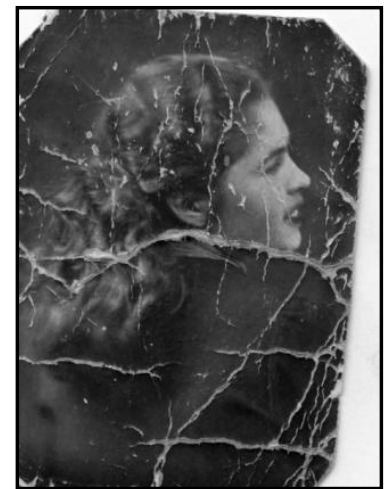

a)

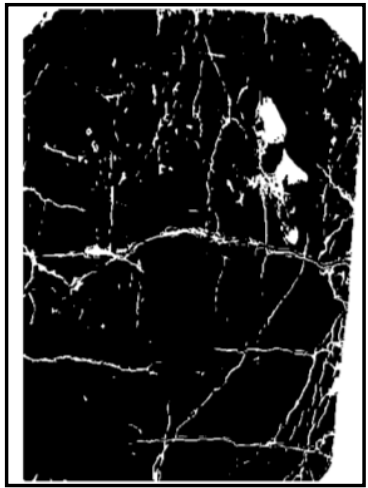

б)

Рисунок 3 - Пример областей получивших большой отклик 
Для сокращения числа ложных срабатываний маска $M_{i, j}$ обрабатывается в скользящем окне $w_{i: i+10, j: j+10}$ размером $10 \times 10$ пикселей, при этом проверяется условие, если четыре крайних пикселя $w_{i, j}, w_{i, j+10}$, $w_{i+10, j}, w_{i+10, j+10}$ равны нулю, то новому результирующему массиву $\bar{M}_{i, j}$ присваивается область содержащая объект, имеющий тонкую структуру из маски $M_{i, j}$. Те области, в которых хотя бы один крайний пиксель не равен нулю в результирующий массив $\bar{M}_{i, j}$ не записываются.

В результате морфологической обработки получается результирующая маска обнаруженных дефектов $\bar{M}_{i, j}$ для восстанавливаемого изображения.

На рисунке 4 представлен пример обработки тестового изображения с целью обнаружения дефектов (а - исходное изображение; б - маска $M_{i, j}$, полученная с параметром $\theta=90^{\circ}$, в - маска $M_{i, j}$, полученная с параметром $\theta=180^{\circ}, \Gamma-$ результирующая маска $\left.\bar{M}_{i, j}\right)$.

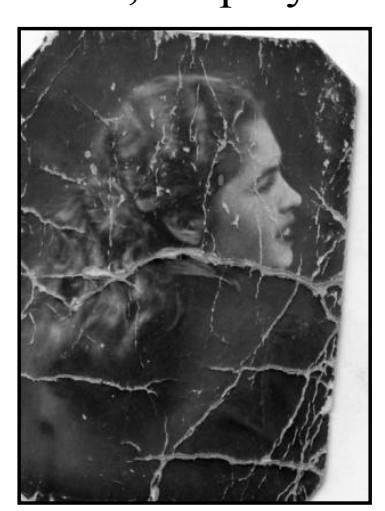

a)

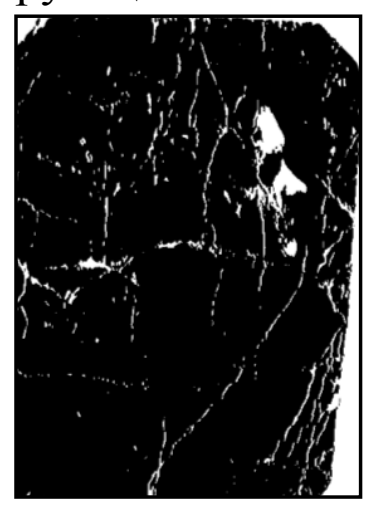

б)

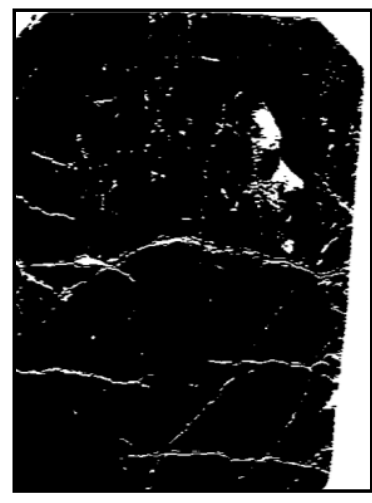

в)

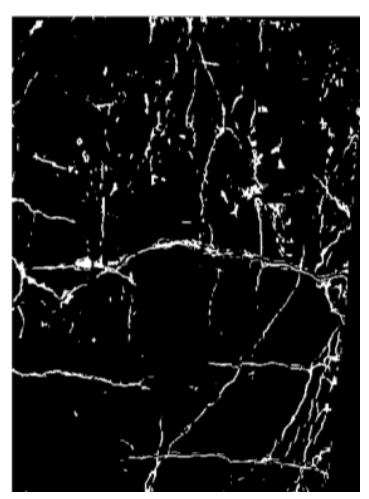

г)

Рисунок 4 - Результат обработки предложенным методом

На рисунках 4б и 4в представлены маски обработанные фильтром Габора с параметром $\theta$ равным 90 и $180^{\circ}$ соответственно. Анализ полученных результатов показывает, что царапины под углом $\approx 45^{\circ}$ на результирующей маске также обнаружены, можно сделать вывод о достаточности двух сверток для обнаружения царапин любых направлений. Следует отметить, что увеличение количества сверток с разными направлениями $\theta$ может привести к увеличению количества ложных срабатываний.

На рисунке 5 представлены две результирующие маски. Маска, изображенная на рисунке $5 б$ получена в результате свертке с ядром, для которого ось нормали функции Габора равна $\theta=90^{\circ}$ и $\theta=180^{\circ}$, а маска, приведенная на рисунке 5в получена в результате свертки с ядром, для которого ось нормали функции Габора равна $\theta=45^{\circ}$ и $\theta=-45^{\circ}$. 


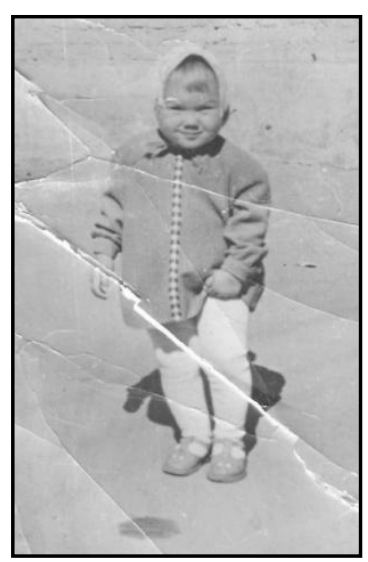

a)

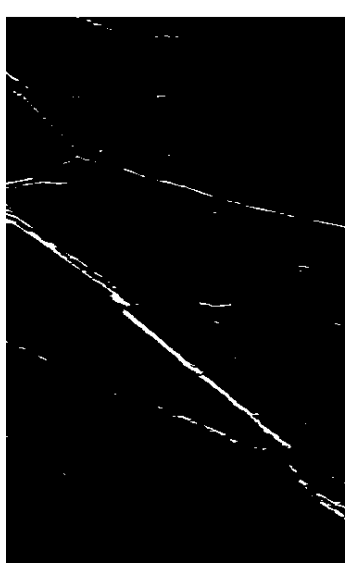

б)

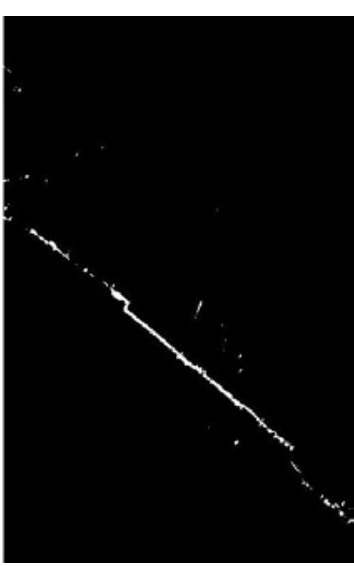

B)

\section{Рисунок 5 - Пример работы предложенного подхода:}

a) - исходное изображение; б) - результирующая маска $\bar{M}_{i, j}$ с параметрами $\theta=90^{\circ}$ и $\theta=180^{\circ}$; в) - результирующая маска $\bar{M}_{i, j} \mathbf{c}$ параметрами $\theta=45^{\circ}$ и $\theta=-45^{\circ}$

Анализ результатов обработки показывает, что маска дефектов, полученная с помощью диагональных ядер имеет незначительные отличия от маски, полученной с помощью ядер $\theta=90^{\circ}$ и $\theta=180^{\circ}$.

В заключении можно сделать следующие выводы:

- Предлагается подход к обнаружению дефектов, представленных в виде трещин на поврежденных изображениях, основанный на анализе свертки изображения с ядром Габора.

- Представлены результаты обработки тестовых изображений.

- Обоснован выбор параметров функции Габора для сокращения количества ложных срабатываний, основанный на геометрических особенностях дефектов.

\section{Литература}

1. Koutsopoulos H. and Downey A. Primitive-based classification of pavement cracking images. Journal of Transportation Engineering, 119(3), 1993.

2. Tanaka N. and Uematsu K. A crack detection method in road surface images using morphology. In Machine Vision Applications, 1998.

3. Giakoumis I., Nikos Nikolaidis N. and Pitas I. Digital image processing techniques for the detection and removal of cracks in digitized paintings. 2005.

4. Oliveira H. and Correia P. Supervised strategies for cracks detection in images of road pavement flexible surfaces. In EUSIPCO, 2008.

5. Chambon S. Detection of points of interest for geodesic contours: 
application on road images for crack detection. International Joint Conference on Computer Vision Theory and Applications, VISAPP, 2011.

6. Воронин В.В. Автоматизированное детектрование дефектов при реконструкции архивных фотодокументов//Научно-технические ведомости СПбГПУ. 2012. № 162.

7. Воронин В.В., Марчук В.И., Гапон Н.В., Сизякин Р.А. Использование метода реконструкции двумерных сигналов при восстановлении архивных фотографий// Успехи современной радиоэлектроники. Зарубежная радиоэлектроника. 2011. № 9.

8. Сизякин Р.А., Воронин В.В., Марчук В.И., Гапон Н.В. Автоматическое обнаружение вертикальных царапин на архивных видеозаписях// Успехи современной радиоэлектроники. Зарубежная радиоэлектроника. 2012. № 6.

9. Сойфер В.А. Методы компьютерной обработки изображений. Физматлит, 2003. - С. 459. 